\title{
Dyakonov-Tamm wave at the planar interface of a chiral sculptured thin film and an isotropic dielectric material
}

\author{
Akhlesh Lakhtakia \\ akhlesh@psu.edu \\ John A. Polo, Jr. \\ polo@edinboro.edu
}

\begin{abstract}
CATMAS-Computational \& Theoretical Materials Sciences Group, Department of Engineering Science and Mechanics, Pennsylvania State University, University Park, PA 16802, USA

Department of Physics and Technology, Edinboro University of Pennsylvania, Edinboro, PA 16444, USA
\end{abstract}

Surface waves, named here as Dyakonov-Tamm waves, can exist at the planar interface of an isotropic dielectric material and a chiral sculptured thin film (STF). Due to the periodic nonhomogeneity of a chiral STF, the range of the refractive index of the isotropic material is smaller but the range of the propagation direction in the interface plane is much larger, in comparison to those for the existence of Dyakonov waves at the planar interface of an isotropic dielectric material and a columnar thin film. Dyakonov-Tamm waves could therefore be detected more easily than Dyakonov waves. [DOI: 10.2971/jeos.2007.07021]

Keywords: Dyakonov wave, sculptured thin film, structural chirality, surface wave, Tamm state, titanium oxide

\section{INTRODUCTION}

Less than two decades ago, Dyakonov [1] theoretically predicted the propagation of a surface wave at the planar interface of an isotropic dielectric material and a positively uniaxial dielectric material with its optic axis wholly parallel to the interface plane. If $\psi$ indicates the angle between the optic axis and the direction of surface-wave propagation, and $n_{s}$ is the refractive index of the isotropic dielectric material, then the Dyakonov wave exists for rather narrow ranges of $\psi$ and $n_{s}$. The consequent significance of Dyakonov waves for optical sensing and waveguiding was recognized thereafter $[2,3]$. Since then, the concept of the Dyakonov wave has been extended to the planar interfaces of isotropic and biaxial dielectric materials [4]. The possibility of the anisotropic material being artificially engineered, either as a photonic crystal with a short period in comparison to the wavelength [5] or as a columnar thin film (CTF) [6], has also emerged. Let us note here that the Dyakonov wave still remains to be experimentally observed, in part due to the narrow range of $\psi$ for its existence [5].

The anisotropic material is taken to be homogeneous in all of the foregoing and other reports on the Dyakonov wave. What if the anisotropic material were to be chosen as periodically nonhomogeneous in a direction normal to the bimaterial interface? This question initiated a research project, the first results of which are being communicated here. Being a natural extension of a CTF, a chiral sculptured thin film (STF) was chosen as the periodically nonhomogeneous anisotropic material [7].

A chiral STF is made by directing a vapor flux in vacuum at an oblique angle onto a rotating substrate. Under suitable conditions, an assembly of parallel nanohelixes of the evaporated species forms, with the helical axes perpendicular to the substrate. An example of a single nanohelix is illustrated in Figure 1 .

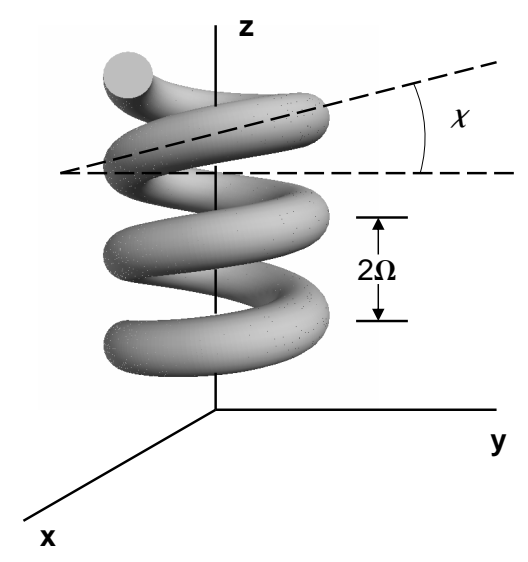

FIG. 1 Geometry of a structurally right-handed helix.

By adjusting deposition parameters, both the pitch $2 \Omega$ and the angle of inclination $\chi \in(0, \pi / 2]$ can be controlled. Each nanohelix, composed of multimolecular clusters with $\sim 3 \mathrm{~nm}$ diameter, is effectively a continuously bent column of $\sim 100$ $\mathrm{nm}$ cross-sectional diameter. Therefore, at visible frequencies and lower, a chiral STF may be regarded as a linear, locally orthorhombic, unidirectionally nonhomogeneous continuum whose relative permittivity dyadic is akin to that of chiral smectic liquid crystals [8].

In formulating the surface-wave-propagation problem on the planar interface of an isotropic, homogeneous, dielectric material and a chiral STF, we adopted a methodology originally developed by Tamm in 1932 for a realistic Kronig-Penney model. Instead of assuming the solid to occupy the entire space, as is commonplace in solid-state physics [9], Tamm assumed the solid to occupy only a half-space. The incorporation of the oft-neglected surface led to the emergence of electronic states 
localized to the surface. Tamm states were experimentally observed in 1990 on the surfaces of superlattices [10], and their optical analogs for superlattices of isotropic materials are being investigated these days [11, 12].

Given the braiding of Dyakonov waves and Tamm states in this communication, we decided to name the surface wave at the planar interface of an isotropic, homogeneous, dielectric material and a chiral STF as the Dyakonov-Tamm wave. Section 2 presents the boundary-value problem and the dispersion equation for the Dyakonov-Tamm wave. Section 3 contains numerical results when the chiral STF is chosen to be made of titanium oxide $[6,13]$. An $\exp (-i \omega t)$ timedependence is implicit, with $\omega$ denoting the angular frequency. The free-space wavenumber, the free-space wavelength, and the intrinsic impedance of free space are denoted by $k_{o}=\omega \sqrt{\epsilon_{o} \mu_{0}}, \lambda_{o}=2 \pi / k_{0}$, and $\eta_{0}=\sqrt{\mu_{o} / \epsilon_{o}}$, respectively, with $\mu_{o}$ and $\epsilon_{o}$ being the permeability and permittivity of free space. Vectors are underlined, dyadics underlined twice; column vectors are underlined and enclosed within square brackets, while matrixes are underlined twice and similarly bracketed. Cartesian unit vectors are identified as $\underline{u}_{x}, \underline{u}_{y}$ and $\underline{u}_{z}$. The dyadics employed in the following sections can be treated as $3 \times 3$ matrixes $[14,15]$.

\section{FORMULATION}

\subsection{Geometry and permittivity}

Let the half-space $z \leq 0$ be occupied by an isotropic, homogeneous, nondissipative, dielectric material of refractive index $n_{s}$. The region $z \geq 0$ is occupied by a chiral STF with unidirectionally nonhomogeneous permittivity dyadic given by [7]

$$
\underline{\underline{\epsilon}}(z)=\epsilon_{o} \underline{\underline{S}}_{z}(z) \cdot \underline{\underline{S}}_{y}(\chi) \cdot \underline{\underline{\epsilon}}_{\mathrm{ref}} \cdot \underline{\underline{S}}_{y}^{T}(\chi) \cdot \underline{\underline{S}}_{z}^{T}(z), \quad z \geq 0,
$$

where the reference relative permittivity dyadic

$$
\underline{\epsilon}_{r e f}=\epsilon_{a} \underline{u}_{z} \underline{u}_{z}+\epsilon_{b} \underline{u}_{x} \underline{u}_{x}+\epsilon_{c} \underline{u}_{y} \underline{u}_{y}
$$

indicates the locally orthorhombic symmetry of the chiral STF. The dyadic function

$$
\begin{aligned}
\underline{S}_{z}(z)= & \cos \left(\frac{\pi z}{\Omega}+\psi\right)\left(\underline{u}_{x} \underline{u}_{x}+\underline{u}_{y} \underline{u}_{y}\right) \\
& +h \sin \left(\frac{\pi z}{\Omega}+\psi\right)\left(\underline{u}_{y} \underline{u}_{x}-\underline{u}_{x} \underline{u}_{y}\right)+\underline{u}_{z} \underline{u}_{z}
\end{aligned}
$$

contains $2 \Omega$ as the structural period, $\psi$ as an angular offset, and $h= \pm 1$ as the handedness parameter. The tilt dyadic

$$
\begin{aligned}
\underline{\underline{S}}_{y}(\chi)= & \left(\underline{u}_{x} \underline{u}_{x}+\underline{u}_{z} \underline{u}_{z}\right) \cos \chi \\
& +\left(\underline{u}_{z} \underline{u}_{x}-\underline{u}_{x} \underline{u}_{z}\right) \sin \chi+\underline{u}_{y} \underline{u}_{y}
\end{aligned}
$$

involves the angle of inclination $\chi$. The superscript ${ }^{T}$ denotes the transpose.

Without loss of generality, we take the Dyakonov-Tamm wave to propagate parallel to the $x$ axis in the plane $z=0$. There is no dependence on the $y$ coordinate, whereas the DyakonovTamm wave must attenuate as $z \rightarrow \pm \infty$.

\subsection{Field representations}

In the region $z \leq 0$, the wave vector may be written as

$$
\underline{k}_{s}=\kappa \underline{u}_{x}-\alpha_{s} \underline{u}_{z},
$$

where

$$
\kappa^{2}+\alpha_{s}^{2}=k_{o}^{2} n_{s}^{2},
$$

$\kappa$ is positive and real-valued for unattenuated propagation along the $x$ axis, and $\operatorname{Im}\left[\alpha_{s}\right]>0$ for attenuation as $z \rightarrow-\infty$. Accordingly, the field phasors in the region $z \leq 0$ may be written as

$$
\underline{E}(\underline{r})=\left[A_{1} \underline{u}_{y}+A_{2}\left(\frac{\alpha_{s}}{k_{o}} \underline{u}_{x}+\frac{\kappa}{k_{o}} \underline{u}_{z}\right)\right] \exp \left(i \underline{k}_{s} \cdot \underline{r}\right), \quad z \leq 0,
$$

and

$$
\underline{\underline{H}}(\underline{r})=\eta_{o}^{-1}\left[A_{1}\left(\frac{\alpha_{s}}{k_{o}} \underline{u}_{x}+\frac{\kappa}{k_{o}} \underline{u}_{z}\right)-A_{2} n_{s}^{2} \underline{u}_{y}\right] \begin{array}{r}
\exp \left(i \underline{k}_{s} \cdot \underline{r}\right), \\
z \leq 0,
\end{array}
$$

where $A_{1}$ and $A_{2}$ are unknown scalars.

The field representation in the region $z \geq 0$ is more complicated. It is appropriate to write

$$
\left.\begin{array}{l}
\underline{E}(\underline{r})=\underline{e}(z) \exp (i \kappa x) \\
\underline{H}(\underline{r})=\underline{h}(z) \exp (i \kappa x)
\end{array}\right\}, \quad z \geq 0,
$$

and create the column vector

$$
[\underline{f}(z)]=\left[\begin{array}{llll}
e_{x}(z) & e_{y}(z) & h_{x}(z) & h_{y}(z)
\end{array}\right]^{T} .
$$

This column vector satisfies the matrix differential equation $[7,16]$

$$
\frac{d}{d z}[\underline{f}(z)]=i\left[\underline{\underline{P}}\left(\frac{\pi z}{\Omega}+\psi, \kappa\right)\right] \cdot[\underline{f}(z)], \quad z>0,
$$

where the $4 \times 4$ matrix

$[\underline{\underline{P}}(\zeta, \kappa)]=$
$\omega\left[\begin{array}{cccc}0 & 0 & 0 & \mu_{0} \\ 0 & 0 & -\mu_{o} & 0 \\ h \epsilon_{o}\left(\epsilon_{c}-\epsilon_{d}\right) \cos \zeta \sin \zeta-\epsilon_{o}\left(\epsilon_{c} \cos ^{2} \zeta+\epsilon_{d} \sin ^{2} \zeta\right) & 0 & 0 \\ \epsilon_{o}\left(\epsilon_{c} \sin ^{2} \zeta+\epsilon_{d} \cos ^{2} \zeta\right)-h \epsilon_{o}\left(\epsilon_{c}-\epsilon_{d}\right) \cos \zeta \sin \zeta & 0 & 0\end{array}\right]$ $+\kappa \frac{\epsilon_{d}\left(\epsilon_{a}-\epsilon_{b}\right)}{\epsilon_{a} \epsilon_{b}} \sin \chi \cos \chi\left[\begin{array}{cccc}\cos \zeta & h \sin \zeta & 0 & 0 \\ 0 & 0 & 0 & 0 \\ 0 & 0 & 0 & -h \sin \zeta \\ 0 & 0 & 0 & \cos \zeta\end{array}\right]$

$$
+\left[\begin{array}{cccc}
0 & 0 & 0 & -\frac{\kappa^{2}}{\omega \epsilon_{o}} \frac{\epsilon_{d}}{\epsilon_{a} \epsilon_{b}} \\
0 & 0 & 0 & 0 \\
0 & \frac{\kappa^{2}}{\omega \mu_{o}} & 0 & 0 \\
0 & 0 & 0 & 0
\end{array}\right]
$$

and

$$
\epsilon_{d}=\frac{\epsilon_{a} \epsilon_{b}}{\epsilon_{a} \cos ^{2} \chi+\epsilon_{b} \sin ^{2} \chi}
$$

Two independent techniques $[17,18]$ exist to solve (11). Either of the two may be harnessed to determine the matrix $[\underline{N}]$ that appears in the relation

$$
[\underline{f}(2 \Omega)]=[\underline{\underline{N}}] \cdot[\underline{f}(0+)]
$$


to characterize the optical response of one period of the chiral STF. By virtue of the Floquet-Lyapunov theorem [19], we can define a matrix $[\underline{Q}]$ such that

$$
[\underline{\underline{N}}]=\exp \{i 2 \Omega[\underline{\underline{Q}}]\}
$$

Both $[\underline{\underline{N}}]$ and $[\underline{\underline{Q}}]$ share the same eigenvectors, and their eigenvalues are also related. Let $[\underline{t}]^{(n)},(n=1,2,3,4)$, be the eigenvector corresponding to the $n$th eigenvalue $\sigma_{n}$ of $[\underline{N}]$; then, the corresponding eigenvalue $\alpha_{n}$ of $[\underline{\underline{Q}}]$ is given by

$$
\alpha_{n}=-i \frac{\ln \sigma_{n}}{2 \Omega}
$$

\subsection{Dispersion equation for Dyakonov-Tamm wave}

For the Dyakonov-Tamm wave to propagate along the $x$ axis, we must ensure that $\operatorname{Im}\left[\alpha_{1,2}\right]>0$, and set

$$
\underline{f}(0+)]=\left[\begin{array}{ll}
{\left[\underline{t}^{(1)}\right.} & {[\underline{t}]^{(2)}}
\end{array}\right] \cdot\left[\begin{array}{l}
B_{1} \\
B_{2}
\end{array}\right],
$$

where $B_{1}$ and $B_{2}$ are unknown scalars; the other two eigenvalues of $[\underline{Q}]$ describe waves that amplify as $z \rightarrow \infty$ and cannot therefore contribute to the Dyakonov-Tamm wave. At the same time,

$$
[\underline{f}(0-)]=\left[\begin{array}{cc}
0 & \frac{\alpha_{s}}{k_{o}} \\
1 & 0 \\
\frac{\alpha_{s}}{k_{o}} \eta_{o}^{-1} & 0 \\
0 & -n_{s}^{2} \eta_{o}^{-1}
\end{array}\right] \cdot\left[\begin{array}{c}
A_{1} \\
A_{2}
\end{array}\right]
$$

by virtue of (7) and (8). Continuity of the tangential components of the electric and magnetic field phasors across the plane $z=0$ requires that

$$
[\underline{f}(0-)]=[\underline{f}(0+)]
$$

which may be rearranged as

$$
\underline{\underline{M}}] \cdot\left[\begin{array}{c}
A_{1} \\
A_{2} \\
B_{1} \\
B_{2}
\end{array}\right]=\left[\begin{array}{l}
0 \\
0 \\
0 \\
0
\end{array}\right] .
$$

For a nontrivial solution, the $4 \times 4$ matrix $[\underline{M}]$ must be singular, so that

$$
\operatorname{det}[\underline{\underline{M}}]=0
$$

is the dispersion equation for the Dyakonov-Tamm wave.

\section{NUMERICAL RESULTS AND DISCUSSION}

Although chiral STFs may be made by evaporating a wide variety of materials [7, Chap. 1], the constitutive parameters of chiral STFs have not been extensively measured. However, the constitutive parameters of certain columnar thin films (CTFs) are known. CTFs are assemblies of nanorods oriented at an
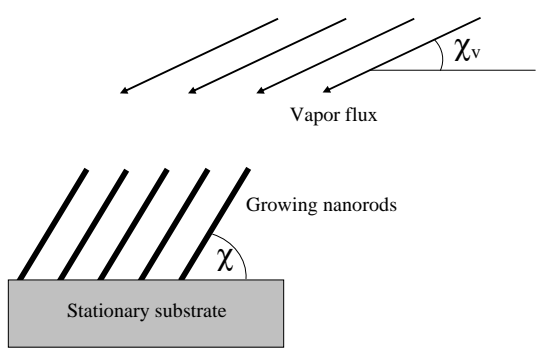

FIC. 2 Schematic of the growth of a columnar thin film. The vapor flux is directed at an angle $\chi_{v}$, whereas nanorods grow at an angle $\chi \geq \chi_{v}$.

angle $\chi$ to the substrate and are produced by directing the vapor at an angle $\chi_{v}$ onto a stationary substrate, as shown in Figure 2; the vapor incidence angle $\chi_{v}$ (in addition to the evaporant species) allows an empirical determination of the constitutive parameters $\epsilon_{a, b, c}$ and $\chi$ [13]. When the substrate is rotated about a normal passing through it at a constant angular velocity of reasonable magnitude, parallel nanohelixes grow instead of parallel nanorods, and a chiral STF is deposited instead of a CTF $[7,20]$. Although the substrate is nonstationary, the functional relationships connecting $\epsilon_{a, b, c}$ and $\chi$ to $\chi_{v}$ for CTFs would substantially apply for chiral STFs, since the vapor incidence angle $\chi_{v}$ remains constant during the deposition of thin films of either kind.

Among the CTFs which have been characterized are those made of titanium oxide, a material important in many practical applications [6]. Empirical relationships have been determined for titanium-oxide CTFs at $\lambda_{o}=633 \mathrm{~nm}$ by Hodgkinson et al. [13] as

$$
\begin{aligned}
& \epsilon_{a}=\left[1.0443+2.7394\left(\frac{\chi_{v}}{\pi / 2}\right)-1.3697\left(\frac{\chi_{v}}{\pi / 2}\right)^{2}\right]^{2}, \\
& \epsilon_{b}=\left[1.6765+1.5649\left(\frac{\chi_{v}}{\pi / 2}\right)-0.7825\left(\frac{\chi_{v}}{\pi / 2}\right)^{2}\right]^{2}, \\
& \epsilon_{c}=\left[1.3586+2.1109\left(\frac{\chi_{v}}{\pi / 2}\right)-1.0554\left(\frac{\chi_{v}}{\pi / 2}\right)^{2}\right]^{2},
\end{aligned}
$$

and

$$
\tan \chi=2.8818 \tan \chi_{v}
$$

where $\chi_{v}$ and $\chi$ are in radian. We must caution that the foregoing expressions are applicable to CTFs produced by one particular experimental apparatus, but may have to be modified for CTFs produced by others on different apparatuses; hence, we used these expressions for the numerical results presented in this section for chiral STFs simply for illustration. Furthermore, we set $h=1, \Omega=197 \mathrm{~nm}$, and $\chi_{v}=7.2^{\circ}$. Following Walker et al. [4] and Polo et al. [6], we left $\psi$ and $n_{s}$ as variable parameters. All numerical results presented in this section were computed for $\lambda_{o}=633 \mathrm{~nm}$.

As mentioned in Section 2, the matrix $[\underline{\underline{N}}]$ can be calculated using two numerical techniques [18]: the piecewise uniform approximation technique and a series technique based on the 
Maclaurin expansion of $[\underline{P}(\zeta, \kappa)]$ with respect to $\zeta$. Both yield the same results, and the piecewise uniform approximation technique was selected for calculations reported here. Basically, the technique consists of subdividing the chiral STF into a series of electrically thin sublayers parallel to the interface, and assuming the dielectric properties to be spatially uniform in each sublayer. The accuracy of this technique depends on the thickness of the sublayers, with thinner ones yielding more accurate results. Based on experience [7, 18], a sublayer thickness of $2 \mathrm{~nm}$ gives reasonable results.

The magnitude of the phase velocity of the Dyakonov-Tamm wave was compared with that of the phase velocity of the electromagnetic wave in the bulk isotropic material. For this purpose, we defined the relative phase speed

$$
\bar{v} \equiv v_{D T} / v_{S},
$$

where $v_{D T}=\omega / \kappa$ is the phase speed of the Dyakonov-Tamm wave and $v_{S}=1 / n_{S} \sqrt{\epsilon_{o} \mu_{o}}$ is the phase speed of the electromagnetic wave in the bulk isotropic material. Figure 3 shows $\bar{v}$ as a function of $\psi$ for several values of $n_{s}$. The phase velocity of the Dyakonov-Tamm wave, like several other surface waves [1]-[6], was found to be lower in magnitude than the phase velocity of the electromagnetic wave in the bulk isotropic material.

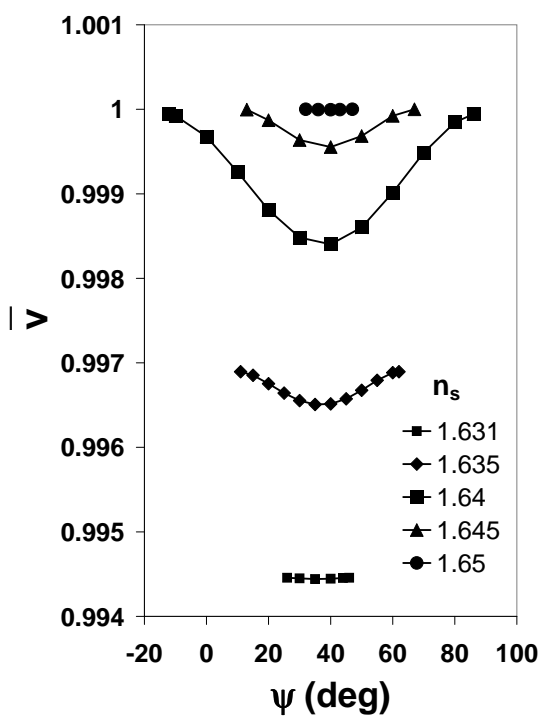

FIG. $3 \bar{v}$ as a function of $\psi$ with $\chi_{v}=7.2^{\circ}$ for $n_{s}=1.631,1.635,1.64,1.645$, and 1.65 .

The minimum and maximum values of $n_{s}$ (1.631 and 1.65, respectively) in Figure 3 represent the approximate limits of the $n_{s}$-range for which the determinantal Eq. (21) representing the boundary conditions between the two material could be solved. Outside this $n_{s}$-range, the Dyakonov-Tamm wave can not exist for the chosen parameters.

Each of the curves in Figure 3 was drawn over the continuous $\psi$-range for which the Dyakonov-Tamm wave was found to exist. The plot in Figure 3 is restricted to $\psi \in\left[-20^{\circ}, 100^{\circ}\right]$. For each value of $\psi$ in this plot (and all remaining plots in this paper), a similar point exists at $\psi+180^{\circ}$ with an identical value of the function; thus, for each value of $n_{s}$, there exist two separate ranges of $\psi \in\left[-180^{\circ}, 180^{\circ}\right]$ over which the
Dyakonov-Tamm waves exist. Of the curves presented, the one for $n_{s}=1.64$, about mid-range in $n_{s}$, has the widest range in $\psi$ with a width $\Delta \psi=98^{\circ}$. The mid-point of the $\psi$-range is $\psi_{m}=37^{\circ}$. As $n_{s}$ approaches either end of the $n_{s}$-range, $\Delta \psi$ diminishes. At the low end of the $n_{S}$-range, $\Delta \psi=51^{\circ}$ when $n_{s}=1.635$, but $\Delta \psi=20^{\circ}$ when $n_{s}=1.631$. At the high end of the $n_{s}$ range, $\Delta \psi=54^{\circ}$ when $n_{s}=1.645$, but $\Delta \psi=15^{\circ}$ when $n_{s}=1.65$. Only a slight variation in $\psi_{m}$, the mid-point of the $\psi$-range, is seen. For $n_{s}=1.631,1.635,1.4,1.645$, and 1.65 , we find $\psi_{m}=36^{\circ}, 36.5^{\circ}, 37^{\circ}, 40^{\circ}$, and $39.5^{\circ}$, respectively. There seems to be a slight increase in $\psi_{m}$ as $n_{s}$ increases. The values of $\psi_{m}$, however, approximate since the end-points of the $\psi$-range were only determined by the last whole degree lying inside the range. This may account for why $\psi_{m}$ at $n_{s}=1.65$ is slightly lower than that at $n_{s}=1.645$.

Every curve in Figure 3 is smooth with a broad minimum in the vicinity of $35^{\circ}$ to $40^{\circ}$ which levels off at both ends of the $\psi$ range. The minimum is deepest for curves representing midrange values of $n_{s}$, while curves at extreme values of $n_{S}$ are nearly flat. As $n_{s}$ decreases, the $\bar{v}$ vs. $\psi$ curve shifts downward.

The confinement of the Dyakonov-Tamm wave to the interface is described by the decay constants which are given by the imaginary part of the two eigenvalues in the chiral STF $\left(\alpha_{1}\right.$ and $\left.\alpha_{2}\right)$ and the single eigenvalue $\alpha_{s}$ in the isotropic dielectric material. We found all three eigenvalues to be purely imaginary, which are shown in Figure 4 as functions of $\psi$ for several values of $n_{s}$.

In Figure 4, typical values of $\operatorname{Im}\left[\alpha_{1}\right]$ are about one order of magnitude larger than $\operatorname{Im}\left[\alpha_{2}\right]$. Every $\operatorname{Im}\left[\alpha_{1}\right]$ vs. $\psi$ curve in Figure $4 \mathrm{a}$ is bell-shaped with a maximum in the vicinity of $\psi=35^{\circ}$ to $40^{\circ}$. The maximum change in $\operatorname{Im}\left[\alpha_{1}\right]$ over the $\psi$ range is greatest for $n_{s}=1.64$, the mid-range of $n_{s}$. At this value of $n_{s}$, the variation in $\operatorname{Im}\left[\alpha_{1}\right]$ is still less than $0.5 \%$. Toward each end of the $n_{s}$-range, the $\operatorname{Im}\left[\alpha_{1}\right]$ vs. $\psi$ curve flattens.

The $\operatorname{Im}\left[\alpha_{2}\right]$ vs. $\psi$ curves in Figure $4 \mathrm{~b}$ show two types of behavior. At the high end of the $n_{s}$-range $\left(n_{s}=1.645,1.65\right)$, the curves are bell-shaped with greater variation occurring at $n_{s}=1.645$ away from the end of the $n_{s}$-range, just as evinced by $\operatorname{Im}\left[\alpha_{1}\right]$. In contrast, in the lower half of the $n_{S}$-range $\left(n_{s}=1.631,1.635,1.64\right)$, the $\operatorname{Im}\left[\alpha_{2}\right]$ vs. $\psi$ curves do not level off at the ends of the respective $\psi$-ranges, but continue to decrease all the way to zero.

The behavior of the decay constant in the isotropic material can be gleaned from the $\operatorname{Im}\left[\alpha_{s}\right]$ vs. $\psi$ curves in Figure $4 \mathrm{c}$. These curves look very similar to those of $\operatorname{Im}\left[\alpha_{2}\right]$ in Figure $4 \mathrm{~b}$. However, the dependence of the shape of the $\operatorname{Im}\left[\alpha_{s}\right]$ vs. $\psi$ curve on $n_{s}$ is opposite that of the $\operatorname{Im}\left[\alpha_{2}\right]$ vs. $\psi$ curve. The curves in Figure $4 \mathrm{c}$ for low values of $n_{s}$ are bell-shaped with $\operatorname{Im}\left[\alpha_{s}\right]$ remaining non-zero over the entire $\psi$-range; but the curves for large values of $n_{s}$ maintain their downward curvature with $\operatorname{Im}\left[\alpha_{s}\right]$ going to zero at both ends of the respective $\psi$-ranges. Thus, the Dyakonov-Tamm wave becomes delocalized from the interface $z=0$ at the limiting values of $n_{s}$; delocalization occurs on the chiral STF side of the interface at low values of $n_{s}$, but on the isotropic substrate side of the interface for high values of $n_{s}$. 

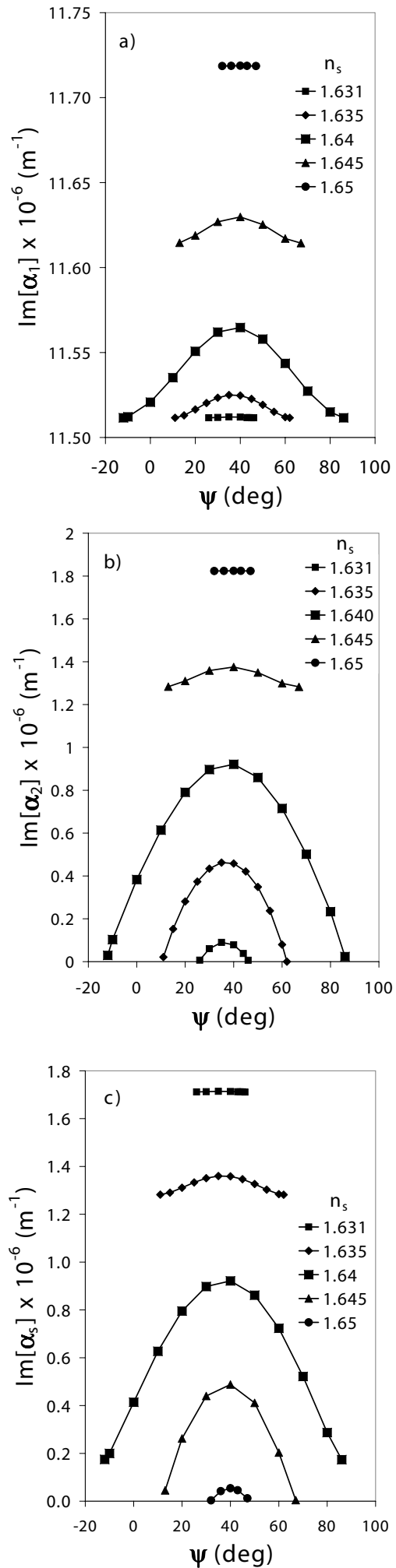

FIG. 4 Decay constants as functions of $\psi$ at $\lambda_{o}=633 \mathrm{~nm}$ for same values of $n_{s}$ and $\chi_{v}$ as in Figure 3. a) $\alpha_{1}$ b) $\alpha_{2}$ c) $\alpha_{s}$.

Similar results were obtained at other values of $\chi_{v}$. As an example, Figure 5 displays $\bar{v}$ when $\chi_{v}=25^{\circ}$.

As in Figure 3, the maximum and minimum values of $n_{s}$ displayed in Figure 5 represent the approximate limits of the range of $n_{s}$ over which surface-wave propagation is possible. The width of the $n_{s}$-range for $\chi_{v}=25^{\circ}$ is roughly half of that obtained for $\chi_{v}=7.2^{\circ}$. The curves for $\chi_{v}=25^{\circ}$ dis-

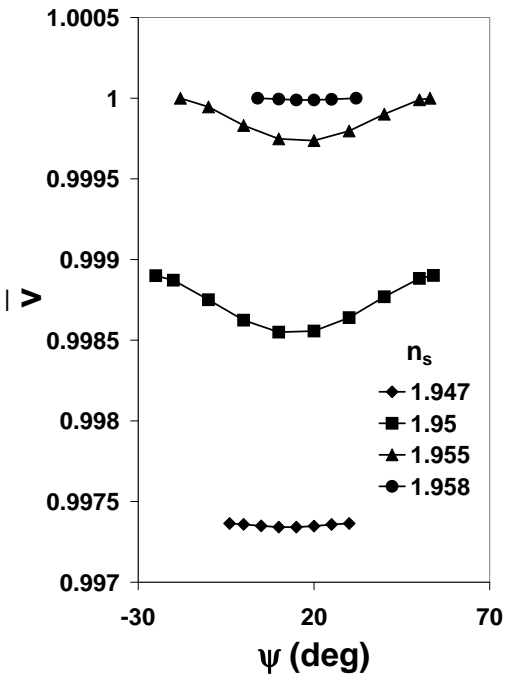

FIG. $5 \bar{v}$ as a function of $\psi$ with $\chi_{v}=25^{\circ}$ for $n_{s}=1.947,1.95,1.955,1.958$.

play the same general shape and trends as for the case of $\chi_{v}=7.2^{\circ}$, with similar values of $\Delta \psi$. However, the minimums have shifted to a lower value of $\psi$ between $15^{\circ}$ and $20^{\circ}$. Now, $\psi_{m}=13^{\circ}, 14.5^{\circ}, 17.5^{\circ}$, and $18^{\circ}$ for $n_{s}=1.947,1.950,1.955$, and 1.958 , respectively. Again, a slight upward drift of $\psi_{m}$ as $n_{s}$ increases can be seen. The figure also shows that the minimum value of $\bar{v}$ is larger for $\chi_{v}=25^{\circ}$ than it is for $\chi_{v}=7.2^{\circ}$. Although not shown, all three decay constants are lower at the higher value of $\chi_{v}$.

Calculations were also made at $\lambda_{o}=533$ and $733 \mathrm{~nm}$, with the assumption of the same constitutive and geometric parameters as at $\lambda_{o}=633 \mathrm{~nm}$. Qualitatively similar conclusions on the width of the ranges of $n_{S}$ and $\psi$ for Dyakonov-Tamm waves were drawn from the numerical results obtained. Furthermore, the minimum value of $\bar{v}$ was found to increase and the maximum values of $\operatorname{Im}\left[\alpha_{1}\right], \operatorname{Im}\left[\alpha_{2}\right]$, and $\operatorname{Im}\left[\alpha_{s}\right]$ were found to decrease, as the ratio $\lambda_{0} / \Omega$ increases.

Additional calculations, not shown here, were made for an identical chiral STF except with structural left-handedness $(h=-1)$. The exact same results were obtained with the lefthanded chiral STF as with the right-handed chiral STF presented in Figures 2-4. It must borne in mind that, by changing $h$ from \pm 1 to $\mp 1$ in (3), we invert not only the structural handedness of the chiral STF but also the sense of rotation brought about by $\psi \neq 0$.

\section{CONCLUDING REMARKS}

To conclude, we examined the phenomenon of surface-wave propagation at the planar interface of an isotropic dielectric material and a chiral sculptured thin film. The boundaryvalue problem was formulated by marrying the usual formalism for the Dyakonov wave at the planar interface of an isotropic dielectric material and a columnar thin film with the methodology for Tamm states in solid-state physics. The solution of the boundary-value problem let us deduce the existence of Dyakonov-Tamm waves. 
In constitutive terms, the major difference between a CTF and a chiral STF is the periodic nonhomogeneity enshrined in $\underline{\underline{S}}_{z}(z)$; in the limit $\Omega \rightarrow \infty$, a nanohelix uncurls into a nanorod, and a chiral STF transmutes into a CTF. However, the distinction between chiral STFs and CTFs is nontrivial, as may be deduced from the Floquet-Lyapunov theorem $[19,16]$. In comparison to the Dyakonov wave localized to the planar interface of an isotropic dielectric material and a CTF [6], we found that the $n_{s}$-range for the existence of a Dyakonov-Tamm wave at the planar interface of an isotropic dielectric material and a chiral STF is smaller. However, the $\psi$-range is much larger in width: in comparison to $\Delta \psi<1^{\circ}$ with CTFs [6] - and $\Delta \psi<5^{\circ}$ with effectively uniaxial, short-period photonic crystals [5] the width of the $\psi$-range is as high as $98^{\circ}$ in Figure 3 with chiral STFs. This implies that Dyakonov-Tamm waves could be detected much more easily than Dyakonov waves.

\section{ACKNOWLEDGMENT}

A.L. gratefully acknowledges a fascinating discussion with Profs. J. Martorell (Universidad de Barcelona) and D.W.L. Sprung (McMaster University). Two anonymous reviewers are thanked for their suggestions.

\section{References}

[1] M. I. D'yakonov, "New type of electromagnetic wave propagating at an interface" Sov. Phys. JETP 67, 714-716 (1988).

[2] L. Torner, J. P. Torres and D. Mihalache, "New type of guided waves in birefringent media" IEEE Photonic. Tech. L. 5, 201-203 (1993).

[3] L. Torner, J. P. Torres, C. Ojeda and D. Mihalache, "Hybrid waves guided by ultrathin films" J. Lightwave Technol. 13, 2027-2033 (1995).

[4] D. B. Walker, E. N. Glytsis and T. K. Gaylord, "Surface mode at isotropic-uniaxial and isotropic-biaxial interfaces" J. Opt. Soc. Am. A $15,248-260$ (1998).

[5] D. Artigas and L. Torner, "Dyakonov surface waves in photonic metamaterials" Phys. Rev. Lett. 94, 013901 (2005).

[6] J. A. Polo, Jr., S. R. Nelatury and A. Lakhtakia, "Propagation of surface waves at the planar interface of a columnar thin film and an isotropic substrate" J. Nanophoton. 1, 013501 (2007).

[7] A. Lakhtakia and R. Messier, Sculptured thin films: Nanoengineered morphology and optics (SPIE Press, Bellingham, WA, USA, 2005).

[8] P.G. de Gennes and J. Prost, The physics of liquid crystals (Clarendon Press, Oxford, United Kingdom, 1993).

[9] C. Kittel, Introduction to solid state physics (Wiley Eastern, New Delhi, India, 1974).

[10] H. Ohno, E. E. Mendez, J. A. Brum, J. M. Hong, F. Agulló-Rueda, L. L. Chang and L. Esaki, "Observation of "Tamm states" in superlattices" Phys. Rev. Lett. 64, 2555-2558 (1990).

[11] J. Martorell, D. W. L. Sprung and G. V. Morozov, "Surface TE waves on 1D photonic crystals" J. Opt. A: Pure Appl. Op. 8, 630-638 (2006).

[12] A. Namdar, I. V. Shadrivov and Y. S. Kivshar, "Backward Tamm states in left-handed metamaterials" Appl. Phys. Lett. 89, 114104 (2006).

[13] I. J. Hodgkinson, Q. h. Wu and J. Hazel, “Empirical equations for the principal refractive indices and column angle of obliquely deposited films of tantalum oxide, titanium oxide, and zirconium oxide" Appl. Optics 37, 2653-2659 (1998).

[14] H. C. Chen, Theory of electromagnetic waves (TechBooks, Fairfax, VA, USA, 1992).

[15] J. Van Bladel, Electromagnetic fields (Hemisphere, Washington, DC, USA, 1985).

[16] A. Lakhtakia and W. S. Weiglhofer, "Further results on light propagation in helicoidal bianisotropic mediums: oblique propagation" P. Roy. Soc. Lond. A Mat. 453, 93-105 (1997); correction: 454, 3275 (1998).

[17] M. Schubert and C. M. Herzinger, "Ellipsometry on anisotropic materials: Bragg conditions and phonons in dielectric helical thin films" Phys. Status Solidi A 188, 1563-1575 (2001).

[18] J. A. Polo, Jr. and A. Lakhtakia, "Comparison of two methods for oblique propagation in helicoidal bianisotropic mediums" 0pt. Commun. 230, 369-386 (2004).

[19] V. A. Yakubovich and V. M. Starzhinskii, Linear differential equations with periodic coefficients (Wiley, New York, NY, USA, 1975).

[20] R. Messier, V. C. Venugopal and P. D. Sunal, "Origin and evolution of sculptured thin films" J. Vac. Sci. Technol. A 18, 1538-1545 (2000). 